\title{
Zum Gebrauch von Idiomen in der Sprache der Politik Eine kontrastive Analyse der Vorkommenshäufigkeit und Struktur von Somatismen in deutschen und kosovarischen Parlamentsdebatten
}

\author{
Milote SADIKU / Vjosa HAMITI ${ }^{1}$
}

\begin{abstract}
On the use of idioms in the language of politics. A contrastive analysis of the frequency and structure of idioms featuring names of body parts in German and Kosovar parliamentary debates

This article deals with the use of idioms featuring the names of body parts in political speech, specifically in parliamentary debates. Its aim is to investigate the frequency of occurrence of idioms featuring the names of body parts. Accordingly, it investigates which names of body parts occur most frequently in German and Kosovar parliamentary debates. A further aim of the study is to examine the communicative-pragmatic performance of idioms featuring the names of body parts in this genre. As is well known, politicians often use idioms with the aim of expressing emotions and attitudes about certain events and thereby creating a degree of closeness to their voters.
\end{abstract}

Keywords: idioms, body parts, political speech, emotions

DOI: $10.15452 /$ StudiaGermanistica.2021.29.0003

\section{Einleitung}

Der vorliegende Beitrag befasst sich mit dem Gebrauch von Phrasemen mit Körperteilbezeichnungen in der Sprache der Politik, genauer in Parlamentsdebatten. Ziel dieses Beitrages ist es, die Vorkommenshäufigkeit von Phrasemen mit Körperteilbezeichnungen zu untersuchen. Dementsprechend wird analysiert, welche Körperteilbezeichnungen am häufigsten in den deutschen und kosovarischen Parlamentsdebatten vorkommen. Im Mittelpunkt der Untersuchung steht auch die Analyse der kommunikativ-pragmatischen Leistung der Somatismen in diesem Texttyp. Wie bekannt ist, verwenden Politiker oft Phraseme mit dem Ziel, Emotionen und Einstellungen über bestimmte Ereignisse zu äußern und dadurch auch eine gewisse Nähe zu ihren Wählern zu schaffen. Als Materialgrundlage

Gleichberechtigte Autorinnen 
dienen dem korpusbezogenen Teil dieser Untersuchung jeweils fünf stenographische Berichte des Deutschen Bundestages und des Kosovarischen Parlaments.

Somatismen zählen zu den zahlreichsten und gebräuchlichsten Phrasemen, sowohl im Deutschen als auch im Albanischen. Aufgrund der hohen Phraseoaktivität von Somatismen und ihrem universellen Charakter wurde entschieden, Phraseme mit Körperteilbezeichnungen zum Gegenstand dieses kontrastiven Beitrags zu machen. ${ }^{2}$ Aufgrund des Untersuchungsgegenstandes dieses Beitrags ergeben sich folgende Fragestellungen:

1. Kommen mehr Phraseme mit Körperteilbezeichnungen in deutschen oder in kosovarischen Parlamentsdebatten vor?

2. Welche Körperteilbezeichnungen sind am zahlreichsten in den deutschen und kosovarischen Parlamentsdebatten vorhanden?

\section{Phraseme in der Sprache der Politik}

In diesem Beitrag wird in Anlehnung an Fleischer (1997:29 ff.), Burger (2015:14) und Wotjak (1992:3) von folgender Begrifflichkeit der Phraseme ausgegangen: Phraseme werden als (relativ) stabile sprachliche Einheiten verstanden, die die Eigenschaften Polylexikalität, Festigkeit (Phraseme im weiteren Sinn) und Idiomatizität (Phraseme im engeren Sinn) aufweisen, die durch Lexikalisierung und Reproduzierbarkeit gekennzeichnet sind, deren oberste Grenze der Satz ist. Im vorliegenden Beitrag wird eine breite Definition des Begriffs Phrasem angenommen, und darunter werden nicht nur idiomatische, sondern auch schwach idiomatische und nicht-idiomatische phraseologische Einheiten zusammengefasst. Die Verwendung des weiten Phraseologiebegriffs bei der Analyse politischer Reden rechtfertigt Elspaß (2007:285) dadurch, dass eine Einschränkung auf Idiome nur einen Bruchteil der phraseologischen Charakteristika politischer Reden offenlegen würde.

Phraseme werden in der einschlägigen Fachliteratur als beliebtes Stilmittel betrachtet, denen eine besondere Rolle in der Textgestaltung zukommt (vgl. Sabban 2007; Stein 2017). Phraseme haben ein besonderes textbildendes Potenzial, eine „textbildende Potenz“(vgl. Wotjak 1992:3). Dieses textbildende Potenzial von Phrasemen ,richtet sich auf die Beziehungen zwischen Phrasemen, ihren Erscheinungsformen und Verwendungsweisen sowie ihren Funktionen im Text" (Sabban 2007:237). Durch ihre Verwendung wird der Text anschaulicher, expressiver und persuasiver. Doch die Realisationen dieser „Potenzen“ hängen vom Gesamtkontext ab und werden durch unterschiedliche Kommunikationsfaktoren bestimmt. (vgl. Fleischer 1997:214).

Phraseme tragen zur Anschaulichkeit und Expressivität bei, und werden oft als rhetorisch-stilistische Mittel in der Sprache der Politik eingesetzt. Der Einsatz von Phrasemen im politischen Sprachgebrauch steigert die informativ-persuasive Funktion der politischen Sprache. Das Augenmerk richtet sich auf die Verwendung von Phrasemen in politischen Textsorten, insbesondere auf politische Reden (vor allem die parlamentarische Debattenrede), oder auch auf die politische Berichterstattung in den Medien (vgl. Stein 2017).

Elspaß (2007:285) hebt hervor, dass eigentlich jeder Diskurs politisch sein kann, und dass nicht alle Aussagen von Politikern als politisch einzustufen seien. Demnach gibt es also keine sogenannte politische Sprache. Jedoch teilen nicht alle diese Ansicht. Bei einer Durchsicht der einschlägigen sprachwissenschaftlichen Forschungsliteratur zum politischen Sprachgebrauch fällt auf, dass Sprache in der Politik vor allem sprachliches Handeln innerhalb der Politik bedeutet. Dieckmann definiert Politik als „staatliches oder auf den Staat bezogenes Reden“ (Dieckmann 1975:29), das zugleich nicht nur das Handeln von Individuen, sondern von Gruppen einschließt (vgl. Dieckmann 2005:13). Die enge Verbindung der Sprache mit der Politik ist markant. Zurecht schreibt Dieck-

Eine weitere Motivation für diese Entscheidung war, dass wir davor Kopf-Phraseme im Albanischen, Bosnischen/Kroatischen/Serbischen und Mazedonischen kontrastiv analysiert haben und unsere Forschungsinteressen über die vorliegende Untersuchung hinaus erweitert haben. 
mann, dass „Handeln nur so lange politisches Handeln ist, als es sprachliches Handeln ist. Wo Politik sprachlos wird, hört Politik auf zu existieren“"(Dieckmann 1975:29).

Das Handlungspotenzial von Sprache, die für die Politik konstitutiv ist und in der sich nur schwer zwischen Reden und Handeln unterscheiden lässt, wird in mehreren Studien hervorgehoben (vgl. hierzu Girnth 2015; Eppler 1992; Heringer 1990 u. a.). Heringers Vorschlag, statt von Sprache in der Politik von Politik in der Sprache zu sprechen, soll ,darauf hinweisen, dass Politik sich in Sprache vollzieht und dass politische Tätigkeit sprachliche Tätigkeit ist“" (Heringer 1990:9).

Im Vergleich zur Alltagssprache lässt sich die Sprache der Politiker als besonders emotiv, symbolhaft und werthaltig kennzeichnen, wobei ihre Persuasionsfunktion besonders hervorgehoben wird (vgl. Bergsdorf 1983:37, Klein 2010:9). Ein weiterer Unterschied zur Alltagssprache ist auch der Gebrauch von politischen Begriffen, die sich im Vergleich zu anderen Sprachbereichen durch ihre normierende bzw. präskriptive Leistungsfähigkeit auszeichnen (vgl. Bergsdorf 1983:46). Diese Begriffe sind laut Bergsdorf ,nicht nur Symbole wie Wörter, die als Namen oder Zeichen für einen Gegenstand, eine Substanz oder eine Handlung stehen. Begriffe sind verdichtete Symbole, die für Zusammenhänge stehen und durch sie bestimmt werden“ (Bergsdorf 1991:22).

Der Gebrauch von Phrasemen in politischen Reden ist von Elspaß $(2000,2007)$ detailliert analysiert worden. Demnach sind Phraseme in der Sprache der Politik keine peripheren Erscheinungen. Rund 10 Prozent der Sprache in Parlamentsreden ist phraseologisch geprägt. Im Profil der „Parlamentsdebatten" dominieren schwach idiomatische oder nicht-idiomatische Verbindungen (Elspaß 2000:266). Dazu zählt er folgende situations- bzw. gesprächsspezifische Verbindungen:

- Konstruktionen mit verba dicendi/sentiendi (ich glaube, ich möchte meinen) und verdeckte Performative (ich sage hier in aller Offenheit),

- einschränkende Prädikationen (meines Erachtens, meiner Meinung nach) und

- Anrede- und Routineformeln (meine Damen und Herren). (Elspaß 2000:267)

Satzgliedwertige idiomatische Phraseme werden weniger häufig verwendet. Stein (2017) geht davon aus, dass sich politische Redner über die Auswirkung prägnanter Phraseologismen in Öffentlichkeit und Medien im Klaren sind und dass sie sich die mit ihrer Verwendung verbundene Wirksamkeit strategisch zunutze machen. In ihren politischen Reden wollen Politiker durch den Einsatz der Phraseme, als rhetorisch-stilistische Mittel, zugleich auch ihre Emotionen und Einstellungen zu bestimmten Sachverhalten andeuten (vgl. Elspaß 2007:289).

\section{Somatismen}

Somatismen beziehen sich auf eine bestimmte Sachgruppe von Phrasemen und gehören zur allgemein gebräuchlichen Lexik. Als Somatismen werden Einheiten bezeichnet, die mindestens eine Komponente enthalten, „die einen menschlichen oder tierischen Körperteil, ein Körperorgan oder eine Körperflüssigkeit“ (Krohn 1994:20) bezeichnet. Phraseme mit einem Körperteil als Komponente werden als sprachliche Universalien betrachtet. ${ }^{3}$ Die Bedeutung der Somatismen lässt sich zum großen Teil voraussagen, ,da der menschliche Körper an sich universell ist und die einzelnen Körperteile dieselben Funktionen erfüllen“ (Valenčič Arh/Pavić Pintarić 2020:53). Somatismen gehören zum ältesten Wortschatz jeder Sprache (vgl. Sadikaj 2010:22) und sind in jeder Sprache reichlich vertreten. Nach Heringer (2020:28) machen Somatismen bis zu 20 Prozent aller festen Wortverbindungen des Deutschen aus. ${ }^{4}$ Somatismen bilden einen besonders brisanten Bereich der Idiomatik (vgl. Schemann 2011:94*).

Bezeichnungen menschlicher Körperteile neigen in hohem Maße dazu, als lexikalische Komponenten von Phraseologien verwendet zu werden (Fleischer 1997:174). Lexeme wie Bein, Arm,

Dazu mehr bei Földes 1985; Krohn 1994; Kahl 2015; Schemann 2011.

Auch nach A. D. Rajchštejn machen Somatismen 15-20\% aller phraseologischen Einheiten des modernen Deutschen aus (zit. nach Krohn 1994: 21). 
Hand, Herz, Kopf, Blut usw. sind besonders phrasem-produktiv und stellen einen essentiellen Bestandteil der Emotions-Phraseme dar. Phraseme mit Körperteilbezeichnungen drücken menschliche emotionale und mentale Fähigkeiten und Einstellungen aus. Phraseme mit Bezeichnungen tierischer Körperteile, wie Schnabel, Kralle, Fell, werden für den Menschen pejorativ verwendet (Heringer 2020: 28). Körperteile werden in vielen Sprachen mit bestimmten Sachverhalten, Tätigkeiten und Eigenschaften assoziiert: Kopf mit Verstand, Herz mit Emotionen, Mund und Zunge mit Sprechen usw. (Krohn 1994:20).

Eine besondere Gruppe von Somatismen verbalisiert den mimischen und gestischen Bereich der Körpersprache der Menschen, wie z.B. den Kopf schütteln, die Nase rümpfen, Achseln zucken. Diese Somatismen nennt Burger Kinegramme. „Mit Kinegrammen wird konventionalisiertes nonverbales Verhalten sprachlich gefasst und kodiert.” (Burger 2015:47). Nach Heringer (2020:28) besteht Körpersprache nicht nur im Agieren mit dem Körper wie Gestik und Mimik. Den Körperteilen werden symbolische Bedeutungen zugeschrieben, welche kognitive Modelle bilden, z.B. Hand steht für ,Handeln', ,Fassen', ,Ergreifen', ,Kontrolle', ,Verantwortung', ,Helfen“ etc. (Heringer 2020:29). Bei einer großen Anzahl von Somatismen steht ein Körperteil für den ganzen Menschen (vgl. Burger 2015:85), wie z.B. heller Kopf oder alb. kokë e madhe ,großer Kopf' . Körperteile als Komponenten der phraseologischen Einheiten werden in solchen Relationen in metonymischer Bedeutung verwendet, indem ein abstrakter Begriff wie Verstand, Klugheit, Dummheit usw. durch einen konkreten Begriff, wie z.B. Kopf, ersetzt wird.

\section{Korpus und Arbeitsmethode}

Für den vorliegenden Beitrag wurden stichprobenartig jeweils fünf stenographische Berichte des Deutschen Bundestages und des Kosovarischen Parlaments von 2020 nach dem Zufallsprinzip ausgewählt, woraus ein Korpus mit Phrasemen mit Körperteilbezeichnungen erhoben wurde. Alle stenographischen Berichte wurden auf der Grundlage des Archivs untersucht, die der Deutsche Bundestag bzw. das Kosovarische Parlament auf ihren offiziellen Webseiten zur Verfügung stellen. Es wurde nach den Körperteilbezeichnungen gesucht, z. B. dt. Hand bzw. alb. dorë, um das Vorkommen der Hand-Phraseme im Text zu überprüfen.

Für die Analyse der Parlamentsdebatten ist es von großer Wichtigkeit, zwischen dem gesprochenen Originaltext und seiner Transkription in den offiziellen gedruckten Versionen des parlamentarischen Verfahrens zu unterscheiden. Da sich stenographische Berichte an Regeln schriftlicher Texte halten, korrigieren sie üblicherweise die paralinguistischen und phrasenbezogenen Merkmale, wie Wiederholungen oder phrasenbezogene Modifikationen usw., die charakteristisch für die gesprochenen Reden sind (vgl. Elspaß 2007:285). Stenographische Berichte ermöglichen zwar „einen recht einfachen Zugriff auf eine repräsentative Auswahl von Parlamentsrednern“, aber sie stellen nicht den Wortlaut der authentischen Parlamentsreden, sondern redigierte Fassungen dar (Elspaß 2000:265; 2007:285), ähnlich verhält es sich auch mit kosovarischen stenographischen Berichten (vgl. Hamiti 2018; Hamiti 2016). Elspaß (2000:267) stellt fest, dass in den stenographischen Berichten der Anteil der Phraseme eindeutig kleiner als in anderen Texttypen bleibt, obwohl Phraseme typisch für die gesprochene Sprache sind.

\subsection{Korpusanalyse}

Im vorliegenden Beitrag wird untersucht, welche Körperteilbezeichnungen als phraseologische Konstituenten in Reden deutscher Politiker und albanischer Politiker im Kosovo am häufigsten vorkommen. Gleichzeitig wird ein zwischensprachlicher Vergleich der Körperteil-Phraseme durchgeführt. Földes betrachtet Phraseme als einen prototypischen Hort des „kulturellen Gedächtnisses“ einer Sprachgemeinschaft (Földes 2007:432). Deutsche und albanische Sprecher leben in jeweils unterschiedlichen Kontexten, geprägt von sich partiell unterscheidender Geschichte, Natur, Kultur und den daraus resultierenden Unterschieden in der Wahrnehmung der Welt. Deshalb ist zu er- 
warten, dass sich in Phrasemen kulturtypische Ereignisse widerspiegeln (vgl. Sadiku 2011:143). Phraseme mit Körperteilbezeichnungen gelten jedoch als sprachliche Universalien, welche auf universellen Gesetzen des menschlichen Denkens basieren. Aus diesem Grund ist nicht stark damit zu rechnen, dass die analysierten Körperteil-Phraseme kulturspezifische Begebenheiten widerspiegeln. In den hier untersuchten Sprachen sind auch phraseologische Einheiten zu finden, die ohne große Differenzen auf der Formseite und in der denotativen Bedeutung verwendet werden.

Die Analyse zeigt, dass die Körperteilbezeichnungen in beiden Sprachen im Hinblick auf die Frequenz unterschiedlich oft vorkommen. Das Korpus, das aus den deutschen und kosovarischen stenographischen Berichten erhoben wurde, besteht insgesamt aus 169 deutschen bzw. 258 albanischen Phrasemen mit Körperteilbezeichnungen. Im Deutschen wurden 17 und im Albanischen 16 Körperteilbezeichnungen mit unterschiedlicher Häufigkeit verwendet. Die quantitativen Ergebnisse, differenziert nach Vorkommenshäufigkeit der Körperteilbezeichnungen in beiden Sprachen, sind in Tabelle 1 zusammengestellt:

\begin{tabular}{|c|c|c|}
\hline Körperteil & Deutsch & Albanisch \\
\hline Hand ,dorë‘ & $50(30 \%)$ & $83(32 \%)$ \\
\hline Auge ,sy' & $47(29 \%)$ & $16(6.2 \%)$ \\
\hline Herz ,zemër' & $17(10.1 \%)$ & $2(0.8 \%)$ \\
\hline Kopf ,kokë‘ & $14(8.3 \%)$ & $10(2.6 \%)$ \\
\hline Rücken ,shpinë‘ & $9(5.3 \%)$ & $5(2 \%)$ \\
\hline Finger, gisht ${ }^{6}$ & $7(4.1 \%)$ & $3(1.2 \%)$ \\
\hline Mund ,gojë‘ & $4(2.4 \%)$ & $5(2 \%)$ \\
\hline Haut, lëkurë‘ & $4(2.4 \%)$ & $2(0.8 \%)$ \\
\hline Fuß ,këmbë‘5 & $3(1.8 \%)$ & $7(2.7 \%)$ \\
\hline Gesicht,fytyrë‘ & $3(1.8 \%)$ & $3(1.2 \%)$ \\
\hline Hals ,qafë‘ & $3(1.8 \%)$ & $10(2.6 \%)$ \\
\hline Haare ,flokë & $3(1.8 \%)$ & 0 \\
\hline Ohr,vesh' & $1(0.6 \%)$ & $14(5.4 \%)$ \\
\hline Nase ,hundë‘ & $1(0.6 \%)$ & $1(0.4 \%)$ \\
\hline Verstand ,mend & $1(0.6 \%)$ & $69(27 \%)$ \\
\hline Blut ,gjak' & $1(0.6 \%)$ & $6(2.3 \%)$ \\
\hline Nerv ,nerv' & $1(0.6 \%)$ & 0 \\
\hline Arm ,krah' & 0 & $6(2.3 \%)$ \\
\hline
\end{tabular}

Tab. 1: Frequenz der Körperteilbezeichnungen in Phrasemen

In dem untersuchten Korpus der Parlamentsdebatten gehört dt. Hand bzw. alb. dorë in beiden Sprachen als Komponente der Phraseme zu den am häufigsten verwendeten Körperteilbezeichnungen, gefolgt von Auge, Herz, Kopf, etc. Mehrere phraseologische Bedeutungen dieser Lexeme sind in beiden Sprachen anzutreffen. Es handelt sich dabei um sprachlich universelle Bedeutungskomponenten. So finden sich Belege in unserem Korpus, bei welchen den Körperteilen symbolische Bedeutungen zugeschrieben werden.

Im Folgenden werden die begrifflichen Bereiche der Körperteilbezeichnungen Hand, Auge, Herz und Kopf, die am häufigsten als Komponenten der Phraseme in unserem Korpus vorkommen, dargestellt:

5 Im Albanischen wird kein lexikalischer Unterschied zwischen Bein und Fuß gemacht. Für beide Konzepte wird das Lexem këmbë/këmba verwendet 
Hand:

Das Lexem Hand steht in Phrasemen in beiden Sprachen unter anderem auch für begriffliche Bereiche wie: ,Autorität', ,Hilfe', ,Macht/Besitz', die in dieser Arbeit durch die Korpusanalyse belegt worden sind:

Autorität: dt. öffentliche Hand - alb. dora e shtetit (wörtl. ,die Hand des Staates')

Eckhard Pols (CDU/CSU): Mit der KfW und der PD - Berater der öffentlichen Hand GmbH haben die Kommunen starke Partner an ihrer Seite, die die Kommunen bei der Umsetzung realistischer Vorhaben unterstützen (Plenarprotokoll 19/150)

Arta Bajralia (VV): Si rrallëherë më parë, qytetarët kanë nevojë për dorën e shtetit në këtë gjendje emergjente, [...] (Transkript vom 25.03.2020) ${ }^{6}$

Hilfe: dt. jmdm. die Hand reichen - alb. i shtriu/i zgjati dorën (dikujt) (wörtl. (jmdm.) die Hand ausstrecken')

Dr. Johann David Wadephul (CDU/CSU): Es gibt also weiterhin eine Notwendigkeit, auch, Herr Kollege, gegen den IS zu kämpfen, und dabei reichen wir eine helfende Hand, dabei unterstützen wir die Koalition, und dabei unterstützen wir den Schlüsselstaat Irak. (Plenarprotokoll 19/154)

Arben Gashi (LDK): Por, ajo çka themi ne këtu dhe për të cilën ne angazhohemi, është që të ndërtojmë një klimë komunikimi, klimë bashkëpunimi, në interes të vendit. Këtë po e kërkojmë. Për këtë angazhohemi dhe për këtë ne jua shtrijmë dorën e bashkëpunimit. (Transkript vom 24.07.2020)7

Macht / Besitz: dt. jmdn., etw. in der Hand haben; in jmds. Hand/Händen sein; etw. liegt in jmds. Händen - alb. e ka në dorë; është në dorën e tij/saj (wörtl. ,(etw./jmdn.) in der Hand haben; es ist in seiner/ihrer Hand')

Dr. Alexander Gauland (AfD): Liebe Kollegen von der FDP, liebe Frau Teuteberg, Sie hatten es ja in der Hand, eine Linksregierung in Thüringen zu verhindern. (Plenarprotokoll 19/150)

Nadine Schön (CDU/CSU):Wir haben es also gemeinsam in der Hand, dass die 20er-Jahre das Jahrzehnt der Frauen werden - in der Politik, in der Arbeitswelt und auch im Bereich der Digitalisierung. Wir haben also vieles zu tun. Unter diesem Zeichen sollte der diesjährige Weltfrauentag stehen. (Plenarprotokoll 19/150)

Albin Kurti (VV): Tjetra, u tha që po dorëzohemi. Unë nuk po jap dorëheqje. Dorëheqja është dorëzim. Kjo është rrëzim. Kurrfarë dorëheqje, kurrfarë dorëzimi s'ka këtu. Është në dorën tuaj a do të rrëzohet Qeveria ose jo. Po as dorëzim, as dorëheqje. [...] Ne japim pa fund fakte për Prokurorinë $q \ddot{e}$ s'vepron as sot e kësaj dite. Po ne s'e kemi në dorë këtë gjë, sepse janë të ndara. (Transkript vom 25.03.2020 $)^{8}$

Auge:

Wie auch Schemann (2011) bekräftigt, ist Auge die komplexeste Komponente der idiomatischen Phraseme. Nach Schemann erscheint das Auge ,in den Phrasemen als Spiegel der Seele und des

6 Jeder albanische Beleg wird in einer Fußnote ins Deutsche übersetzt. Dabei geht es lediglich um den Inhalt der Reden und nicht um die sprachlichen Besonderheiten, die Bestandteil dieser Analyse sind.

,Arta Bajralia (VV): Wie selten zuvor brauchen die Bürger in diesem Ausnahmezustand die Hand des Staates, [...]

7 ,Arben Gashi (LDK): Aber das, was wir hier sagen und wofür wir uns einsetzen, ist es, dass wir im Interesse des Landes ein Kommunikationsklima, ein Zusammenarbeitsklima aufbauen. Das ist es, was wir verlangen. Dafür setzen wir uns ein und reichen dafür die Hand der Zusammenarbeit.

8 ,Albin Kurti (VV): Als nächstes wurde uns gesagt, dass wir aufgeben. Ich resigniere nicht. Resignation ist Aufgeben. Das ist ein Absturz. Hier gibt es keine Resignation, kein Aufgeben. Es liegt in Ihrer Hand, ob die Regierung gestürzt wird oder nicht. Doch, weder Unterwerfung noch Resignation. [...] Wir liefern endlose Fakten für die Staatsanwaltschaft, die auch bis heute nicht handelt. Aber wir haben das nicht in unseren Händen, weil sie getrennt sind. ‘ 
physischen Befindens, als Organ zum (konkreten) Sehen und als ,Instrument' zum geistigen Sehen und Verstehen." (Schemann 2011:102). In den untersuchten Belegen wurden unter anderem folgende begriffliche Bereiche in beiden Sprachen mit der Komponente Auge in Verbindung gebracht:

Erkenntnis: dt. jmdm., sich etw. vor Augen führen/halten:

Martin Hess (AfD): Spätestens als sich das Virus in Italien auszubreiten anfing, hätten Sie Einreisesperren verhängen müssen. Stattdessen haben Sie einen Abschiebestopp nach Italien verhängt. Diese Maßnahme hat allen Wählern wieder einmal klar vor Augen geführt: Ihre Politik der offenen Grenzen und der Weltoffenheit ohne Rücksicht auf den Schaden für unser Land [...] hat für Sie Vorrang vor dem Schutz der deutschen Bevölkerung. Das ist nicht nur leichtsinnig, sondern auch verantwortungslos und inakzeptabel. (Plenarprotokoll 19/153)

Detlef Seif (CDU/CSU): Diejenigen, die behaupten, dass das EU-Türkei-Abkommen tot sei, sollten sich vor Augen halten: In letzter Konsequenz würden wir alle Flüchtlinge, die sich zurzeit in der Türkei aufhalten, im Stich lassen, und wir würden große zusätzliche Migrationsströme in Bewegung setzen. (Plenarprotokoll 19/152)

Hoffnung: alb. drejtoj sytë (kah dikush a diçka) (wörtl. ,die Augen richten [auf jmdn. oder etw.]')

Fikrim Damka (KDTP): Duke pasur parasysh se momentalisht si institucione dhe si shoqëri në çfarë situate alarmante gjendemi [...], ku qytetarët tanimë $i$ kanë drejtuar sytë kah ne, kah përfaqësuesit e tyre legjitimë duke mos pasur asnjë ndihmë të vetme nga institucionet tona, ku po përballen me mungesë të ushqimit, mjeteve higjienike, dorëzave dhe maskave mbrojtëse, kanë ngelur në mëshirën e Zotit dhe nënës natyrë. (Transkript vom 25.03.2020)

Rexhep Selimi (VV): Pra, kërkojnë rrëzimin e Qeverisë kur Bota po vazhdon sërish të përballet me këtë pandemi si problem tashmë global. Kur qytetarët e Kosovës i kanë sytë kah Qeveria, kah ministrat, kah doktorët, kah policët, sërisht LDK-ja nuk ka zgjedhur aleatë tjetër përveç kundërshtarëve të kësaj qeverie. (Transkript vom 25.03.2020) ${ }^{10}$

Herz:

Das Lexem Herz, das mit Emotionen in Verbindung gebracht wird, hat eine viel höhere Frequenz im deutschen Korpus (17 Belege) als im albanischen (2 Belege). Das Ausdrücken der Emotionen scheint den deutschen Politikern wichtiger zu sein als den Kosovaren. In folgenden Belegen wird die Emotion Schmerz zum Ausdruck gebracht:

Katrin Göring-Eckardt (BÜNDNIS 90/DIE GRÜNEN): Die Auswirkungen der Covid-19-Krise bei unseren Nachbarn in Italien und Spanien sind schockierend, und die Nachrichten brechen mir - ich glaube, uns allen-, das Herz. Es beschämt mich als Europäerin, dass unsere erste Reaktion war, die Grenzen zu schließen. (Plenarprotokoll 19/154)

Andreas Jung (CDU/CSU): Es ist keine normale Debatte, so wie es eben eine ganz außergewöhnliche Situation in unserem Land ist. Ich will es so sagen: Es trifft uns ins Herz, wenn wir erleben, wie Kinder weinen, weil sie nicht mit ihren Freunden spielen können, wenn Großeltern ihre Enkel nicht sehen können, [...]. Es trifft uns ins Mark. (Plenarprotokoll 19/154)

9 ,Fikrim Damka (KDTP): Angesichts der Tatsache, dass wir uns derzeit als Institutionen und als Gesellschaft in einer sehr alarmierenden Situation befinden [...], wo die Bürger bereits ihre Augen auf uns gerichtet haben, auf ihre legitimen Vertreter, weil sie keine einzige Hilfe von unseren Institutionen haben, und mit dem Mangel an Nahrungsmitteln, Hygieneartikeln, Handschuhen und Schutzmasken konfrontiert sind, sind Gott und Mutter Natur ausgeliefert geblieben.')

10 ,Rexhep Selimi (VV): Sie fordern also den Sturz der Regierung, wenn die Welt wieder mit dieser Pandemie als einem bereits globalen Problem konfrontiert ist. Wenn die Bürger des Kosovo ihre Augen auf die Regierung, die Minister, die Ärzte, die Polizei richten, hat sich die LDK wiederum keine anderen Verbündeten als die Gegner dieser Regierung ausgesucht.' 
Kopf:

Das Lexem Kopf steht in Phrasemen für den Verstand, das Denken. In den Kopf-Phrasemen wird Kopf als von wesentlicher Bedeutung für jeden Menschen dargestellt, dessen Grundfunktion das Denken ist, und der mental den Menschen steuert und leitet. Dem „Lexikon der Redensarten“ von Müller (2005:328-329) zufolge, ist der Kopf Träger der wichtigsten Sinnesorgane und Sitz des Verstandes. Diese zugrunde liegende Metaphorik ist also kognitiv begründet. Außerdem wird die Komponente Kopf im Deutschen und Albanischen in metonymischer Bedeutung mit Leben in Verbindung gebracht. Diese Bedeutung kommt auch in albanischen Korpusbelegen vor, wie z.B. alb. e shpëtoi kokën (e tij) ,seinen Kopf retten', e pagoi me kokë ,jmd. den Kopf kosten':

Agon Batusha (VV): Ju ofendoni qytetarin dhe inteligjencën e tij sa herë që tentoni të shitni dokrra për antiamerikanizëm e atdhedashuri si shkak për inicimin e mocionit, po ku ka më antiamerikanizëm e antikombëtarizëm se sa të vidhet dhe të zhvatet pasuria nacionale, të shkatërrohet prona publike, mbi të giitha të falet tokë dhe të shkatërrohet shteti nga frika për të shpëtuar kokën nga një gjykatë $q \ddot{e}$ vetë e krijuan. (Transkript vom 25.03.2020) ${ }^{11}$

Saranda Bogujevci (VV): Me gjithë presionin, ajo nuk pranoi të japë dorëheqje, "le të më shkarkojnë, se unë nuk dorëzohem, nuk ka pazare në kurriz të popullit tim e të Kosovës, e paguaj jo me pozitë, por edhe me kokën time për të mirën e vendit tim”, më thoshte. (Transkript vom 25.03.2020) ${ }^{12}$

Die Lexeme dorë ,Hand', mend, Verstand' und vesh ,Ohr' im Albanischen zeigen eine hohe Verwendungsfrequenz. Diese nominalen Komponenten als Prädikatsteil der verbalen festen Wortverbindungen bilden mit dem Verb eine semantische Einheit, die sich syntaktisch wie Einzellexeme verhalten: heq dorë (wörtl. ,eine Hand entfernen') ,zurücktreten, aufgeben'; marr vesh (wörtl. ,Ohr nehmen'), erfahren, verstehen, herausfinden', s'merr vesh (wörtl. ,Ohr nicht nehmen') , nicht gehor-

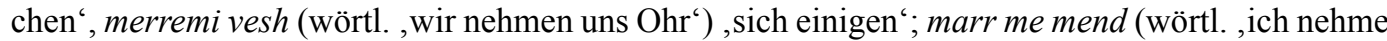
es mit Verstand'), denken, vermuten', mban mend (wörtl. ,es im Verstand behalten'), sich erinnern', (ia) mbush mendjen (wörtl. ,den Verstand füllen (jmdm.)') ,überzeugen'. Diese hohe Frequenz lässt sich dadurch erklären, dass in der gesprochenen Sprache fast ausschließlich die verbalen festen Wortverbindungen und nicht ihre pendanten Ein-Wort-Lexeme verwendet werden, und dass manche Bedeutungsvarianten dieser Wortverbindungen sogar keine Einzellexem-Entsprechung haben.

Die satzgliedwertigen idiomatischen und satzwertigen Phraseme werden sparsam verwendet und gezielt an zentralen Redestellen eingesetzt, denn nur so können sie ihre stilistisch-expressive Wirkung entfalten (Elspaß 2000:268, 275). Bei einer sehr heißen Debatte vom 25.03.2020 im kosovarischen Parlament über den Misstrauensantrag, der gegenüber der damaligen Regierung eingebracht wurde, erwähnten die Politiker sehr oft den letzten Krieg in Kosovo (1999) und die Kriegsopfer, sowie ihren eigenen Beitrag zur Befreiung des Landes. Dabei wurden auch einige idiomatische Wortverbindungen verwendet, die primär an den albanischen Kulturraum gebunden und somit ohne Kontextualisierung schwer in andere Sprachen übertragbar sind. Es handelt sich um einige Idiome mit der Komponente gjak,Blut', in denen das Phänomen der albanischen Blutrache reflektiert wird, wie z.B. e fali gjakun (wörtl. ,Blut verschenken'), sich nicht rächen'; e laj me gjak (wörtl. ,(etw.) mit Blut waschen"), jmd. das Leben kosten'. ${ }^{13}$

11 ,Agon Batusha (VV): Sie beleidigen den Bürger und seine Intelligenz, immer wenn Sie versuchen, Quatsch für Antiamerikanismus und Patriotismus als Begründung für die Initiierung des Misstrauensantrags zu verkaufen. Aber wo gibt es mehr Antiamerikanismus und Antinationalismus, als Volksvermögen zu stehlen und zu plündern, öffentliches Eigentum zu zerstören, vor allem Land zu verschenken und den Staat zu zerstören, aus Angst den Kopf vor einem selbst geschaffenen Gericht zu retten.

12 ,Saranda Bogujevci (VV): Trotz des Drucks weigerte sie sich zurückzutreten, „sie sollen mich entlassen, denn ich gebe nicht auf, es gibt keine Verhandlungen auf Kosten meines Volkes und des Kosovo, ich zahle es nicht mit der Stelle, sondern auch mit meinem Kopf zum Wohle meines Landes“, sagte sie mir.

13 Diese besonderen sprach- und kulturspezifischen Phraseologismen des Albanischen erfordern kulturelles und geschichtliches Hintergrundwissen, denn sie sind mit dem Thema der albanischen Blutrache verbunden, die noch bis Ende des 20. Jahrhunderts nach dem tausendjährigen Kodex in der albanischen Lebensweise gültig war. Sie stammen aus der münd- 
Saranda Bogujevci (VV): [...] Të nderuar deputetë, para se të ngrini dorën për ta votuar mocionin e rrëzimit e Qeverisë në kohë kur po rrezikohemi nga një armik i padukshëm, ndaluni dhe mendoni pak për peshën e vendimit që do ta merrni. Kujdes, se si do ta mbani mend në .histori! [...] Gjakun e familjarëve të mi nuk e fal! E, këtu duhet bashkangjitur edhe unë se gjakun e familjarëve të mi as unë nuk e fal. Edhe tri ditë është përvjetori i familjes, ku janë vrarë më 28 mars, e në këtë kohë ka shumë e shumë familje të tjera që po i kujtojnë të dashurit e tyre në këtë kohë të vështirë. (Transkript vom 25.03.2020 $)^{14}$

Daut Haradinaj (AAK): S'e kam as fije problemi atë punë se kush është ai e kush është ky, se këtë vend e kemi të shenjtë, këtë vend e kemi larë me gjak e pallavra e ligjërata, patriotizëm, dikush që ka vallëzuar poshtë e lartë, nuk ka nevojë të më kallëzojë. Faleminderit! (Transkript vom 25.03.2020) ${ }^{15}$

Auch das folgende vollidiomatische Phrasem des Albanischen e heq qafe (wörtl. sich ,etw./jmdn. vom Hals schaffen), etwas/jmdn. loswerden' wird in der zentralen Redestelle des kosovarischen Premierministers Kurti verwendet. Durch das wiederholte Aufgreifen des Idioms wird sein Wirkungspotenzial entfaltet. Es handelt sich bei diesem Idiom um eine verbale Wendung. Z.B.

\begin{abstract}
Albin Kurti (VV): Unë jam më i bindur se kurrë se marrëveshje të gatshme ka. Praktikisht këtë ma ka konfirmuar edhe Presidenti në takim që kisha. [...] Sepse a e di çka, ma përsëriti ai mua tri herë: hiqe kryeministër taksën, shkojmë bashkë në Uashington, e bëjmë marrëveshjen, e heqim qafe Serbinë përgjithmonë. Tha: „, [...] Shkojmë bashkë atje, e heqim Serbinë qafe njëherë e përgjithmonë”. A po kuptoni? Pra nuk është ideja me heq tarifën për të filluar negociatat. Ideja është me heq tarifën për të publikuar marrëveshjen. [...] Këtu ishte kundërshtimi që kisha unë me presidentin. Ai thoshte, bëjmë marrëveshje me Serbinë dhe e heqim qafe përgjithmonë. Por Serbia ka marrëveshje me Bosnjën, por s'po mund ta heqë qafe. Varet çfarë marrëveshje. Forma nuk shpjegon gjithçka. Fakti që ke marrëveshje nuk do të thotë që e hoqe qafe Serbinë. Ka marrëveshje të keqe që të rëndohet qafa prej saj. (Transkript vom 25.03.2020) ${ }^{16}$
\end{abstract}

In ihren politischen Reden modifizieren Politiker die Phraseme oft, denn dadurch wollen sie den semantischen Mehrwert der Phraseme erhöhen und eine bestimmte stilistische Wirkung erreichen (vgl; Elspaß 2007:289). Auch in unserem Korpus wurden einige Belege mit modifizierten Phrasemen

lichen Volkssprache, die an einer kulturellen, historischen Gegebenheit aus der nationalen Überlieferung gebunden sind. Diese albanische Lebensweise wurde im Kanun von Lekë Dukagjini ausführlich dargestellt.

14 ,Saranda Bogujevci (VV): Geehrte Abgeordnete, bevor Sie Ihre Hand heben, um über den Misstrauensantrag zum Sturz der Regierung abzustimmen, während wir von einem unsichtbaren Feind gefährdet werden, halten Sie inne und denken Sie ein wenig über das Gewicht der Entscheidung nach, die Sie treffen werden. Passen Sie auf, wie Sie sich in der Geschichte daran erinnern werden! [...] Das Blut meiner Familienmitglieder verschenke ich nicht!" Nun, ich muss mich auch daran anschließen, da ich auch das Blut meiner Familienmitglieder nicht verschenke. Nach drei Tagen ist der Jahrestag der Familie, als sie am 28. März ermordet wurde, und zu dieser Zeit gibt es sehr viele andere Familien, die in dieser schweren Zeit ihrer Lieben gedenken.

15 ,Daut Haradinaj (AAK): Ich habe nicht einmal ein Problem damit, wer der eine und wer der andere ist, dass wir diesen Ort heilig haben, dass wir diesen Ort mit Blut und Unsinn und Geschwätz gewaschen haben. Niemand, der auf und ab getanzt hat, soll mir über Patriotismus erzählen. Dankeschön!‘

16 ,Albin Kurti (VV): Ich bin mehr denn je davon überzeugt, dass es eine fertige Vereinbarung gibt. Praktisch wurde mir dies vom Präsidenten beim gemeinsamen Treffen bestätigt. [...] Weil, weißt du was, wiederholte er es mir gegenüber dreimal: Premierminister, schaff die Steuer ab und lass uns zusammen nach Washington gehen, um die Vereinbarung zu treffen, um uns Serbien für immer vom Hals zu schaffen. Er sagte: „,...] Wir gehen zusammen dorthin, wir schaffen uns Serbien für alle Male vom Hals. "Verstehen Sie? Es ist also nicht die Idee, die Steuer abzuschaffen damit wir Verhandlungen aufnehmen. Die Idee ist, die Steuer abzuschaffen, damit man die Vereinbarung bekannt macht. [...] Hier lag der Widerspruch, den ich mit dem Präsidenten hatte. Er sagte, wir treffen eine Vereinbarung mit Serbien und schaffen uns Serbien für alle Male vom Hals. Aber, Serbien hat eine Vereinbarung mit Bosnien, doch Bosnien kann sich Serbien nicht vom Hals schaffen. Es kommt drauf an, was das für eine Vereinbarung ist. Die Form erklärt nicht alles. Die Tatsache, dass du eine Vereinbarung hast, bedeutet nicht, dass du dir Serbien vom Hals geschafft hast. Es gibt schlechte Vereinbarungen, wovon dein Hals schwerer wird. ‘ 
gefunden. So z.B. wird das Phrasem aus den Augen, aus dem Sinn, ${ }^{17}$ wen man nicht mehr sieht, den vergisst man leicht, zu dem reißt der Kontakt ab' als in den Augen, in den Sinn modifiziert. Die Bedeutung des modifizierten Phrasems wird mit dem darauffolgenden Satz erklärt: „Plötzlich bringen die Bilder von der türkischen Grenze das Problem wieder ins Bewusstsein“"

Dr. Gottfried Curio (AfD): Sehr geehrter Herr Präsident! In die Augen, in den Sinn: Plötzlich bringen die Bilder von der türkischen Grenze das Problem wieder ins Bewusstsein: die permanente illegale Migration, die Verweigerung des Grenzschutzes. (Plenarprotokoll 19/152)

Eine weitere Modifikation kommt im folgenden Beleg vor, wobei die Komponente Zorn des Phrasems jmdm. die Zornesröte ins Gesicht treiben (geh.) ,jmdn. sehr zornig machen“ durch Lexem Scham ersetzt wurde:

Kathrin Vogler (DIE LINKE): Was wir doch im Mittelmeer wirklich brauchen, meine Damen und Herren, das ist zivile Seenotrettung. Es muss Ihnen doch die Schamesröte ins Gesicht treiben, dass die Kirchen Geld für ein Rettungsschiff sammeln, [...] während die Bundeswehr im Mittelmeer nicht vorhandene Terroristen sucht. Bekämpfen Sie endlich die Fluchtursachen! Stoppen Sie den Waffenhandel, und retten Sie Menschenleben! Wir haben Platz! (Plenarprotokoll 19/153)

\section{Zusammenfassung}

Ziel des vorliegenden Beitrags war es, die Frequenz sowie die kommunikativ-pragmatische Leistung der Somatismen in der Sprache der Politik, genauer in deutschen und kosovarischen Parlamentsdebatten, zu untersuchen. Als Materialgrundlage der Untersuchung dienten jeweils fünf stenographische Berichte des Deutschen Bundestages und des Kosovarischen Parlaments. Die Analyse der deutschen und kosovarischen stenographischen Berichte ergab, dass die Körperteilbezeichnungen in beiden Sprachen im Hinblick auf die Häufigkeit unterschiedlich vorkommen. Im Deutschen konnten wir insgesamt 169 und im Albanischen 258 Phraseme mit Körperteilbezeichnungen belegen. Im Deutschen wurden 17 und im Albanischen 16 Körperteilbezeichnungen mit unterschiedlicher Häufigkeit verwendet. Bedeutungstechnisch ähnelt sich die Bedeutung in beiden Sprachen zu einem Großteil. 15 Körperteile kommen dabei in beiden Sprachen vor, was sich durch den hochgradig universellen Charakter der Körperteilbezeichnungen in vielen Sprachen erklären lässt. In beiden Sprachen handelt es sich vorwiegend um schwach idiomatische oder nicht-idiomatische Verbindungen und sehr wenige idiomatische Phraseme. Im untersuchten Korpus der beiden Sprachen finden sich Belege, wo die Körperteile mit folgenden begrifflichen Bereichen in Verbindung gebracht werden:

- Hand: mit Autorität, Hilfe, Macht / Besitz;

- Auge: mit Erkenntnis, Hoffnung;

- Kopf: mit Verstand, Denken, Leben usw.

\section{Literaturverzeichnis}

Bergsdorf, Wolfgang (1983): Herrschaft und Sprache: Studie zur politischen Terminologie der Bundesrepublik Deutschland. Pfullingen.

BergSdorf, Wolfgang (1991): Zur Entwicklung der Sprache der amtlichen Politik in der Bundesrepublik Deutschland. In: Liedtke, Frank / Wengeier, Martin / Böke, Karin (Hrsg.): Begriffe besetzen. Strategien des Sprachgebrauchs in der Politik. Opladen, S. 19-33.

Burger, Harald (2015): Phraseologie. Eine Einführung am Beispiel des Deutschen. Berlin.

17 Albanische Äquivalente dieses Phrasems ist: larg sysh, larg zemrës (wörtl. 'weit weg aus den Augen, weit weg aus dem Herz'). 
Dieckmann, Walther (1975): Sprache in der Politik. Einführung in die Pragmatik und Semantik der politischen Sprache. Heidelberg.

Dieckmann, Walther (2005): Deutsch: politisch - politische Sprache im Gefüge des Deutschen. In: KiLIAN, Jörg (Hrsg.): Sprache und Politik. Deutsch im demokratischen Staat. Mannheim; Leipzig; Wien; Zürich, S. 11-30.

DUDEN (2013): Redewendungen. Wörterbuch der deutschen Idiomatik. 11. Aufl. Berlin; Mannheim; Zürich.

DUDEN (2015): Deutsches Universalwörterbuch. Berlin.

ELSPASS, Stephan (2000): Phraseologie im deutschen Parlamentarismus. Zu historischen Entwicklungen im Sprachverhalten politischer Redner. In: Burkhard, Armin / PAPE, Kornelia (Hrsg.): Sprache des deutschen Parlamentarismus. Studien zu 150 Jahren parlamentarischer Kommunikation. Wiesbaden, S. 261-287.

ElsPass, Stephan (2007): Phrasemes in political speech. In: Burger, Harald / Dobrovol'skiJ, Dmitrij / KüHn, Peter / Norrick, Neal R. (Hrsg.): Phraseologie. Ein internationales Handbuch der zeitgenössischen Forschung. 1. Halbband. Berlin; New York, S. 284-292.

EpPler, Erhard (1992): Kavalleriepferde beim Hornsignal. Die Krise der Politik im Spiegel der Sprache. Frankfurt am Main.

FleisCHER, Wolfgang (1997): Phraseologie der deutschen Gegenwartssprache. Tübingen.

FöldEs, Csaba (1985): Über die somatischen Phraseologismen der deutschen, russischen und ungarischen Sprache. Versuch einer konfrontativen Analyse. In: Germanistisches Jahrbuch DDR-UVR. Hrsg. vom Deutschlektorat beim Kultur- und Informationszentrum der DDR in Budapest, S. 18-40

GiRnTh, Heiko (2015): Sprache und Sprachverwendung in der Politik : Eine Einführung in die linguistische Analyse öffentlich-politischer Kommunikation. Berlin; Boston.

Hаміті, Vjosa (2016): Gjuha shqipe në Kuvendin e Republikës së Kosovës. In: Seminari i 35-të Ndërkombëtar për Gjuhën, Letërsinë dhe Kulturën Shqiptare. Prishtinë, S.145-156.

Hаміті, Vjosa (2018): Propagierte Einheit. Der parlamentarische Sprachgebrauch in Albanien und Kosovo. In: Jusufi, Lumnije (Hrsg.): The Potentiality of Pluricentrism Albanian Case Studies and Beyond. Wiesbaden, S. 155-174.

Heringer, Hans Jürgen (1990): „Ich gebe Ihnen mein Ehrenwort.“ Politik - Sprache - Moral. München.

HERINGER, Hans Jürgen (2020): Idiomatik. Eine Einführung. Brey.

KaHL, Stephanie (2015): Kontrastive Analyse zu phraseologischen Somatismen im Deutschen und Italienischen. Magdeburg.

KLeIN, Josef (2010): Politische Sprachstrategien - dargestellt an schweizerischen, deutschen und US-amerikanischen Beispielen. In: Roth, Kersten S. / DürscheID, Christa (Hrsg.): Wahl der Wörter - Wahl der Waffen? Sprache und Politik in der Schweiz. Bremen, S. 19-35.

KroHn, Karin (1994): Hand und Fuß. Eine kontrastive Analyse von Phraseologismen im Deutschen und Schwedischen. Göteburg.

RAJChŠTEIN, A. D (1980): O postavlenii frazeologičeskich sistem, In: Innostrannyje jazyki v škole, 4, S. 8-17.

Sabban, Annette (2007): Textbildende Potenzen von Phrasemen. In: Burger, Harald / Dobrovol'skis, Dmitrij / KÜHN, Peter / Norrick, Neal R. (Hrsg.): Phraseologie. Ein internationales Handbuch der zeitgenössischen Forschung. 1. Halbband. Berlin; New York, S. 237-253.

SADIKAJ, Sonila (2010): Metaphorische Konzepte in somatischen Phraseologismen des Deutschen und Albanischen. Eine kontrastive Untersuchung anhand von Herz- und Hand-Somatismen. Würzburg. Zugänglich unter: https://opus.bibliothek.uni-wuerzburg.de/opus4-wuerzburg/frontdoor/deliver/index/docId/4244/file/WespA_9.pdf [05.12.2021]

SADIKU, Milote (2011): Deutsche und albanische Phraseologismen mit Tierbezeichnungen im Kontrast. In: Linguistik online, 47, 3. Bern, S. 141-152. Zugänglich unter: https://bop.unibe.ch/ linguistik-online/article/view/368 
Schemann, Hans (2011): Deutsche Idiomatik. Die deutschen Redewendungen im Kontext. Berlin; Boston; Göttingen.

Stein, Stephan (2017): Phraseologismen. In: Roth, Kersten S. / Wengeler, Martin / Ziem, Alexander (Hrsg.): Handbuch Sprache in Politik und Gesellschaft. Berlin; Boston.

Thomai, Jani (1999): Fjalor frazeologjik i gjuhës shqipe. Tiranë.

Valenčič Arh, Urška / Pavić Pintarić, Anita (2020). Somatismen als Kodierungsmittel in Konfliktdialogen. In: Slavia Centralis, 13, 1. Maribor, S. 52-68.

WotJAK, Barbara (1992): Verbale Phraseolexeme in System und Text. Tübingen. 